\title{
Radiographic and clinical outcomes of surgical treatment of Kümmell's disease with thoracolumbar kyphosis: a minimal two-year follow-up
}

Hao Cheng ${ }^{1,2}$, Guo-dong Wang ${ }^{3}$, Tao Li ${ }^{3}$, Xiao-yang Liu ${ }^{3}$ and Jian-min Sun ${ }^{1 *}$

\begin{abstract}
Background: Up to now in the surgical treatment of Kümmell's disease combined with thoracolumbar kyphosis, little research has focused on the evaluation of the imaging and clinical outcomes of restoring the normal alignment and sagittal balance of the spine. This study aimed to evaluate the short to mid-term radiographic and clinical outcomes in the treatment of Kümmell's disease with thoracolumbar kyphosis.

Methods: From February 2016 to May 2018, 30 cases of Kümmell's disease with thoracolumbar kyphosis were divided into group A and B according to whether the kyphosis was combined with neurological deficits. All of the cases underwent surgical treatment to regain the normal spinal alignment and sagittal balance. The radiographic outcomes and clinical outcomes of the cases were retrospectively evaluated. The sagittal imaging parameters including sagittal vertebral axis (SVA),thoracic kyphosis (TK),thoracolumbar kyphosis (TLK),lumbar lordosis (LL),pelvic incidence (PI),pelvic tilt (PT),and sacral slope (SS) before operation,immediately after operation,and the last follow-up of each case were measured and evaluated. The clinical results included the Oswestry Disability Index (ODI) and the Numerical Rating Scale (NRS) of the two groups. Statistical software SPSS21.0 was used to analyze the data.

Results: In group A: Mean SVA before operation was $75 \mathrm{~mm}$ and $26.7 \mathrm{~mm}$ at the final postoperative evaluation $(P=0.000)$; Mean TLK before operation was $39^{\circ}$, and $7.1^{\circ}$ at the final postoperative evaluation $(P=0.000)$; Mean NRS before operation was 4.7, compared with 0.9 at the final postoperative evaluation $(P=0.000)$. In group B: Mean preoperative SVA was $62.5 \mathrm{~mm}$ and decreases to $30.7 \mathrm{~mm}$ at the final postoperative evaluation $(P=0.000)$; Mean TLK before operation was $33^{\circ}$, and $9.7^{\circ} 2$ years post-operation $(P=0.000)$; Mean NRS prior to surgery was 4.0 , and 0.8 at the last follow-up evaluation $(P=0.000$ ). The improvement of the NRS scores of groups $A$ and $B$ was related to the improvement of the cobb angle $(P=0.020)$; $(P=0.009)$ respectively.
\end{abstract}

Conclusion: In the treatment of Kümmell's disease with thoracolumbar kyphosis,to restore the normal alignment and sagittal balance can obtain a satisfactory radiographic and clinical short and medium-term effects.

Keywords: Osteoporotic vertebral compression fracture, Kümmell's disease, Kyphosis, Imbalance, Spine, Pain,treatment

\footnotetext{
* Correspondence: Spine2000@msn.cn

'Department of Spine Surgery, Shandong Provincial Hospital, Cheeloo

College of Medicine, Shandong University, Jinan, Shandong, China

Full list of author information is available at the end of the article
}

C C The Author(s). 2021 Open Access This article is licensed under a Creative Commons Attribution 4.0 International License, which permits use, sharing, adaptation, distribution and reproduction in any medium or format, as long as you give appropriate credit to the original author(s) and the source, provide a link to the Creative Commons licence, and indicate if changes were made. The images or other third party material in this article are included in the article's Creative Commons licence, unless indicated otherwise in a credit line to the material. If material is not included in the article's Creative Commons licence and your intended use is not permitted by statutory regulation or exceeds the permitted use, you will need to obtain permission directly from the copyright holder. To view a copy of this licence, visit http://creativecommons.org/licenses/by/4.0/ The Creative Commons Public Domain Dedication waiver (http://creativecommons.org/publicdomain/zero/1.0/) applies to the data made available in this article, unless otherwise stated in a credit line to the data. 


\section{Background}

Since Kümmell's disease was first proposed by Hermann Kümmell in 1895,it has been gradually recognized and understood by spinal surgeons [1]. With the aging of the population, Kümmell's disease,as a complication of osteoporotic vertebral compression fracture (OVCF) [2-5], also shows increased incidence $[6,7]$. At present, Kümmell's disease is defined as ischemic necrosis of the vertebral body after minor spinal injury $[5,6,8]$. As the disease progresses, the injured vertebra gradually collapse, and tend to become wedge shaped due to bone necrosis, followed by kyphosis deformity of the spine.

The treatment of Kümmell's disease is still challenging for most spinal surgeons. One reason is that there are several different stages of progression of Kümmell's disease, but the choice of surgical methods for cases of different stages is still controversial [3-5, 9-11]. Especially for the patients with kyphosis,most opinions agree that in the treatment of kyphosis with neurological deficits the injured vertebrae should be removed by posterior vertebral column resection (VCR) to relieve spinal cord compression, then kyphosis should be corrected and spinal stability should be reconstructed [12-17], so as to obtain better clinical effects. However, as for kyphosis with no neurological impairment, most of the literature focus on eliminating the unstable factors of injured vertebra, so as to relieve local chronic and refractory lower back pain $[4,18-21]$.

Previous theories of Kümmell's disease suggest that there are two different types of pain, acute pain secondary to a fresh fracture at an early stage, and a chronic low back pain that develops gradually after a plateau of weeks or months [1-3, 6-8]. As for the causes of chronic pain in the later stage, most of the literature indicates that it is due to bone necrosis, fracture nonunion, and instability caused by local micro-motion of the injured vertebra $[6,7,9,11$, 14]. As the understanding of the spine sagittal balance has deepened, it has gradually been realized that a good spine sagittal balance could help the lower back muscles do a minimal work to maintain a balanced posture [22, 23]. Conversely, the advancement of the gravity line caused by long-term kyphotic deformity of the spine will not only change the stress of the corresponding segment on the intervertebral disc, facet joints, and other tissue structures, but also will further accelerate the degeneration of the spine leading to the appearance of discogenic low back pain. At the same time, long-term kyphosis will cause strain and degeneration of the back extensors, which will further aggravate the sagittal imbalance [24, 25]. It has been reported that patients with chronic kyphotic deformities or sagittal imbalance have a higher proportion of long-term refractory pain in the back [26, 27].

Kümmell's disease occurs frequently in thoracic and lumbar segments [28]. And kyphosis secondary in the above segments often leads to the sagittal imbalance, which can easily lead to further aggravation of existing low back pain. Although more and more spine surgeons have begun to pay attention to sagittal spinal balance in the past 10 years, there are few studies published in the literature about surgical treatment of thoracolumbar kyphosis.

A fact that almost all spine surgeons accept is that the normal physiological angle of the thoracolumbar segment is $0^{\circ}[29,30]$. And it has also been reported in the literature that patients with thoracolumbar sagittal imbalance usually experienced a significant improvement in low back pain after corrective surgery $[26,31]$. Considering the factors above for the further evaluation of postoperative imaging and clinical effects of Kümmell's disease with thoracolumbar kyphosis, Shandong Provincial Hospital conducted this retrospective study.

\section{Methods}

\section{Ethics}

This retrospective study was approved by the Medical Ethics Committee of Shandong Provincial Hospital and was conducted in accordance with the guidelines of the Declaration of Helsinki. Written informed consent was obtained from each patient.

\section{Selection of patients and clinical and imaging evaluation criteria}

From February 2016 to May 2018, 30 cases of Kümmell's disease with thoracolumbar kyphosis who underwent surgical treatment in our department were recruited into this study. All patients were divided into two groups according to whether there were neurological deficits. And the imaging parameters and clinical evaluation indexes of patients before operation, after operation and at the last follow-up were collected and analysed.

Group A included 14 cases of kyphosis with neurological deficits,including 12 females and 2 males, with an average age of $66.8 \pm 7.5$ years. The mean $\mathrm{T}$ value of bone mineral density (BMD) was $-3.3 \pm 1.4$. As for the neurological function, according to the American Spinal Injury Association (ASIA) impairment scale, 2 cases were classified as B, 5 cases as $C$ and 7 cases as D.

Group B included 16 cases of kyphosis with no neurological deficits, including 13 females and 3 males, with an average age of $64.3 \pm 7.7$ years; the mean $\mathrm{T}$ value of BMD was $-3.6 \pm 0.4$. 


\section{Inclusion criteria \\ Group A:}

1. Meet the diagnostic criteria for Kümmell's disease, $\mathrm{T}$ value of $\mathrm{BMD}<-2.5$.

2. After conservative treatment for more than 3 months,refractory low back pain still exists.

3. Kyphosis appeared gradually,and it continued to progress. Neurological deficits appeared gradually and aggravated slowly with the progression of kyphosis.

4. Single segment kyphosis with neurological deficits was graded as B-D, according to ASIA impairment scale.

Group B: In line with the 1-2 criteria above, thoracolumbar kyphosis is associated with sagittal global/local parameter abnormalities or sagittal imbalance without neurological deficits.

\section{Exclusion criteria}

1. Kümmell's disease without kyphosis.

2. Severe cardiovascular and cerebrovascular diseases;Diabetes mellitus and other contraindications.

3. Multiple segmental osteoporotic fractures.

4. Kümmell's disease with old spinal fractures of other segment(s).

5. Patients with lumbar disc herniation,ankylosing spondylitis, spinal tuberculosis, lumbar spondylolisthesis and spinal tumors.

6. Patients who had undergone spinal surgery or vertebroplasty before.

\section{Imaging evaluation parameters}

SVA, Cobb angle, TK,TLK,LL,PI,PT,SS.

\section{Clinical evaluation indexes}

The ODI, NRS, and ASIA grades and complications were all recorded, including infection, deep vein thrombosis (DVT) of lower limbs, cerebrospinal fluid (CSF) leakage, subsidence of internal implants, broken screws and rods, pseudoarthrosis etc. Since it was inconvenient to perform ODI and NRS assessment immediately after surgery, only preoperative assessment and final assessment were performed. Moreover, the recovery of neurological function was relatively slow, so the neurological function of preoperative and final assessment were performed.

\section{Surgical procedure}

Group A: Under general anesthesia and electrophysiological monitoring, the patient was prone on the operating bed. A posterior median incision was performed centering on the injured vertebra. Firstly,pedicle screws were placed in the two upper and two lower vertebrae with the injured vertebra as the center and each screw was strengthened with bone cement. If the injured vertebra was a thoracic vertebra, the proximal ends of bilateral ribs were removed for about $5 \mathrm{~cm}$ then the spinous process and the lower $2 / 3$ of the lamina of the upper vertebrae of the injured vertebra were excised. Next the spinous process and lamina of the injured vertebra were excised to expose the spinal canal, protecting the dura and nerve roots. And then the injured vertebra and its upper and lower intervertebral discs were excised. After that, a C-shaped cage made of polyetheretherketone of appropriate size was selected, and autologous bone grains were inserted and placed between the upper and lower end plates of adjacent vertebral bodies. Afterwards the two pre-bent connecting rods were installed, and the cantilever beam technology was used for orthopedic and pressurized locking. During the process of VCR, a temporary rod was used to maintain the spinal stability. Finally, after the drainage tube was placed in the incision, and the incision was closed with layer by layer sutures (Fig. 1).

Group B: After taking the same procedures of general anesthesia,electrophysiological monitoring as in group A, patients in group B were also exposed with a posterior midline incision. After that,pedicle screws were placed in the two upper and two lower vertebrae on the both sides of the intervertebral space which closed to the collapsed endplate of the injured vertebrae, and the screws were cemented too. Next, the bilateral inferior articular processes of the upper vertebra of the injured vertebra and the bilateral superior articular processes of the injured vertebra were resected. The lower 2/3 lamina of the upper vertebra and the upper 1/3 lamina of the injured vertebra were resected, and the ligamentum flavum was also resected. To protect the nerve roots of both sides, discectomy was performed from the lateral of the dura, and the intervertebral space was released thoroughly. Soon after the upper and lower endplates of the intervertebral space were removed (bone-disc-bone osteotomy), autologous bone granulation grafting in the intervertebral space was performed, and finally the kyphosis was corrected. After the drainage tube was placed in the incision, the incision was closed with layer by layer sutures too (Fig. 2).

Post operation careful attention was paid to the prevention of infection and complications such as deep venous thrombosis of lower extremities.

The drainage tube was removed 3-5 days after the operation, and the patient got out of bed as soon as possible under the protection of braces. Support protection of the brace spanned 3 months. Patients in both groups 

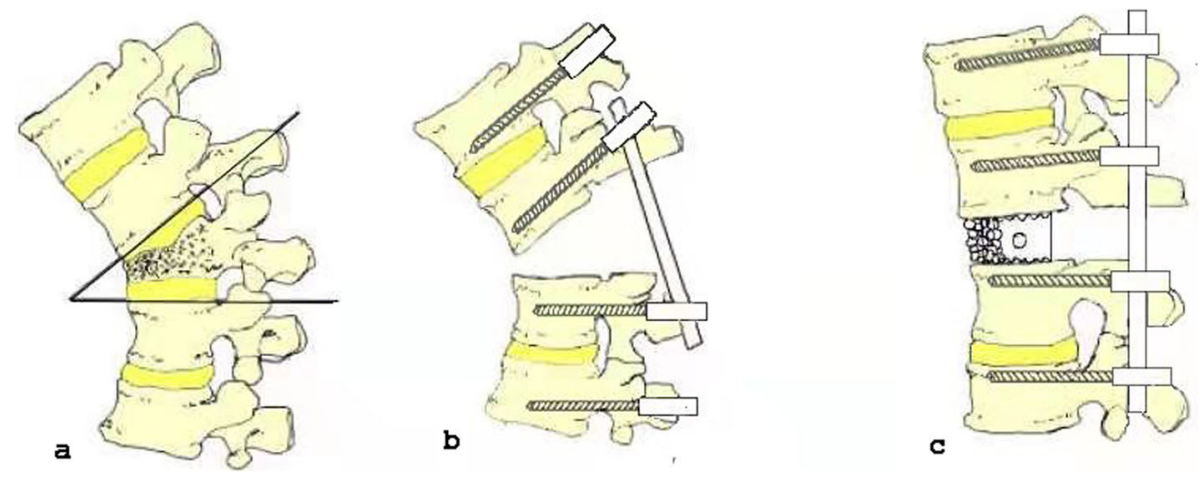

Fig. 1 Surgical diagram of group A

were treated for osteoporosis after surgical treatment according to the current consensus procedures.

\section{Statistical methods}

Statistical software SPSS21.0 was used for statistical analysis of the above statistical data. The comparison of general data and postoperative and final imaging parameters between the two groups was performed by independent sample $T$ test. One-way ANOVA was used to compare preoperative and postoperative imaging parameters and the last imaging parameters. Paired $\mathrm{T}$ test was used to compare ODI and NRS results in groups. Pearson correlation was used to analyze the correlation between the improvement of NRS and the change of sagittal parameters. Setting $P<0.05$ was statistically significant.

\section{Results}

Group A: The average operative time was $336 \pm 60 \mathrm{~min}$, the average operative blood loss was $1125 \pm 769 \mathrm{ml}$, and the average follow-up time was 34.2 months (range 24-50 months). There were 2 cases of CSF leakage, 2 cases of incision infection, 1 case of implant subsidence, 1 case of pseudoarthrosis, and 1 case of Proximal Junctional Kyphosis (PJK). In the last follow-up,there was 1 case of Grade C, 3 cases of Grade D and 10 cases of Grade E with Neurological function of ASIA impairment scale, with an average increase of 1.8 levels compared with preoperative level (Table 1).

Group B: the average operation time was $270 \pm 48$ min, the average blood loss was $442 \pm 159 \mathrm{ml}$, and the average follow-up time was 38.7 months (range 36-53 months), there was 1 case of CSF leakage, 2 cases of incision infection, 1 case of DVT of lower extremity, and 1 case of pseudoarthrosis. No neurological deficits appeared in group B after operation and at the last follow-up (Table 1).

\section{Imaging evaluation}

In this study, the normal range of SVA was selected as \pm $40 \mathrm{~mm}$.

In Group A:

SVA, Cobb Angle, TLK, PT, ODI and NRS were significantly different among the preoperative,

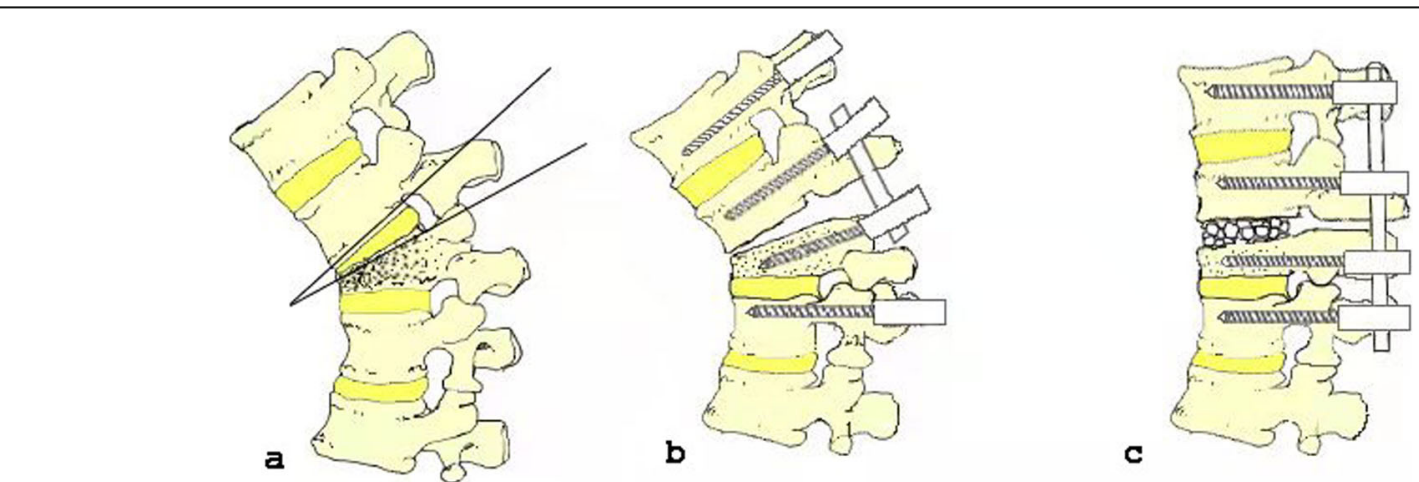

Fig. 2 Surgical diagram of group B 
Table 1 Comparison of general data before and after operation between group $A$ and $B$

\begin{tabular}{llll}
\hline Parameters & Group A & Group B & P value \\
\hline Age (years) & $66.8 \pm 7.5$ & $64.3 \pm 7.7$ & 0.440 \\
Male & 2 & 3 & \\
Female & 12 & 13 & 0.743 \\
T value of BMD & $-3.3 \pm 1.4$ & $-3.6 \pm 0.4$ & 0.628 \\
Operation time (minutes) & $336 \pm 60$ & $270 \pm 48$ & 0.010 \\
Blood loss (ml) & $1125 \pm 769.3$ & $441.7 \pm 159.3$ & 0.007 \\
Preoperative SVA (mm) & $75.0 \pm 39.2$ & $62.5 \pm 26.6$ & 0.386 \\
PreopCobb angle $\left(^{\circ}\right)$ & $28.2 \pm 6.2$ & $26.5 \pm 7.3$ & 0.560 \\
Preop ODI & $59 \% \pm 9$ & $44 \% \pm 8$ & 0.000 \\
Preop NRS & $4.7 \pm 0.9$ & $4.0 \pm 1.1$ & 0.225 \\
CSF leakage & 2 & 1 & \\
Incision infection & 2 & 2 & \\
Implant subsidence & 1 & 0 & \\
Pseudoarthrosis & 1 & 1 & \\
PJK & 1 & 0 & \\
DVT & 0 & 1 & \\
\hline
\end{tabular}

postoperative and last follow-up $(P<0.05)$ (Table 2) (Fig. 3). There was no statistically significant difference in TK, LL, and SS $(P>0.05)$ (Table 2).

In Group B:

SVA, Cobb Angle, TLK, ODI and NRS were significantly different among the preoperative, postoperative and final follow-up $(P<0.05)$ (Table 3$)$ (Fig. 4). There was no significant difference in TK, LL, PT, and SS $(P>$ 0.05) (Table 3).

Comparison of postoperative imaging results between group A and B: Cobb angle, and PT difference were statistically significant $(P<0.05)$ (Table 4$)$. There were no statistically significant differences in SVA, TK, TLK, LL, and SS $(P>0.05)$ (Table 4$)$.
Comparison of imaging and clinical results at the last follow-up between group A and B: Cobb angle, TLK, PT, SS showed statistically significant differences $(P<0.05)$ (Table 5). There was no significant difference in SVA, TK, LL, ODI, and NRS $(P>0.05)$ (Table 5).

The result of correlation analysis showed that the improvement of the NRS of both groups was related to the improvement of the cobb angle $(P<0.05)$. In group A, the improvement of NRS was also positively correlated with the change of PT but negatively correlated with the change of SS $(P<0.05)$ (Tables 6,7$)$.

\section{Discussion}

The authors believe that the balance of the thoracolumbar segment is of great clinical significance for the global balance of the spine, and the restoration of sagittal balance is a key factor in improving quality of life. Therefore, in the treatment of Kümmell's disease with thoracolumbar kyphosis, to ensure the stabilization of the spine and to relieve the compression to the spinal cord, additional attention should be paid to the restoration of normal thoracolumbar alignment and the correction of sagittal imbalance, so as to avoid or reduce the later refractory low back pain, and to further improve the patient outcomes. Therefore, during the operation, not only the thoracolumbar kyphosis angle of the two groups was restored to the normal range, but also the global balance of the spine sagittal plane was restored. According to Schwab's classification standard for posterior spinal osteotomy [32], VCR belongs to grade $\mathrm{V}$ osteotomy. In group A, after a standard VCR was performed, and with the help of the posterior pedicle internal fixation system, it was relatively easy to correct thoracolumbar kyphosis, to restore normal thoracolumbar alignment and to correct sagittal imbalance. Postoperative results and the last follow-up showed a satisfactory recovery of sagittal parameters.

Table 2 Comparison of data before and after operation and the last follow-up in group A

\begin{tabular}{lllll}
\hline Parameter & Before operation & After operation & Final follow-up & $\boldsymbol{p}$ value \\
\hline SVA $(\mathrm{mm})$ & $75.0 \pm 39.2$ & $25.1 \pm 17.1$ & $26.7 \pm 16.2$ & 0.000 \\
Cobb angle $\left(^{(}\right)$ & $28.2 \pm 6.2$ & $6.4 \pm 5.0$ & $7.3 \pm 5.3$ & 0.000 \\
TK $\left(^{\circ}\right)$ & $34.3 \pm 11.7$ & $37.2 \pm 10.3$ & $39.0 \pm 21.1$ & 0.783 \\
TLK $\left(^{\circ}\right)$ & $39.0 \pm 21.1$ & $5.7 \pm 3.3$ & $7.1 \pm 3.1$ & 0.000 \\
LL $\left(^{\circ}\right)$ & $45.0 \pm 14.3$ & $34.4 \pm 7.5$ & $38.4 \pm 5.0$ & 0.066 \\
PT $\left(^{\circ}\right)$ & $29.0 \pm 6.8$ & $20.6 \pm 7.7$ & $22.6 \pm 8.2$ & 0.048 \\
SS $\left(^{\circ}\right)$ & $28.2 \pm 9.9$ & $35.6 \pm 9.9$ & $36.5 \pm 9.2$ & 0.129 \\
ODI & $59 \% \pm 9$ & & $23 \% \pm 5$ & 0.000 \\
NRS & $4.7 \pm 0.9$ & & $0.9 \pm 0.3$ & 0.000 \\
\hline
\end{tabular}




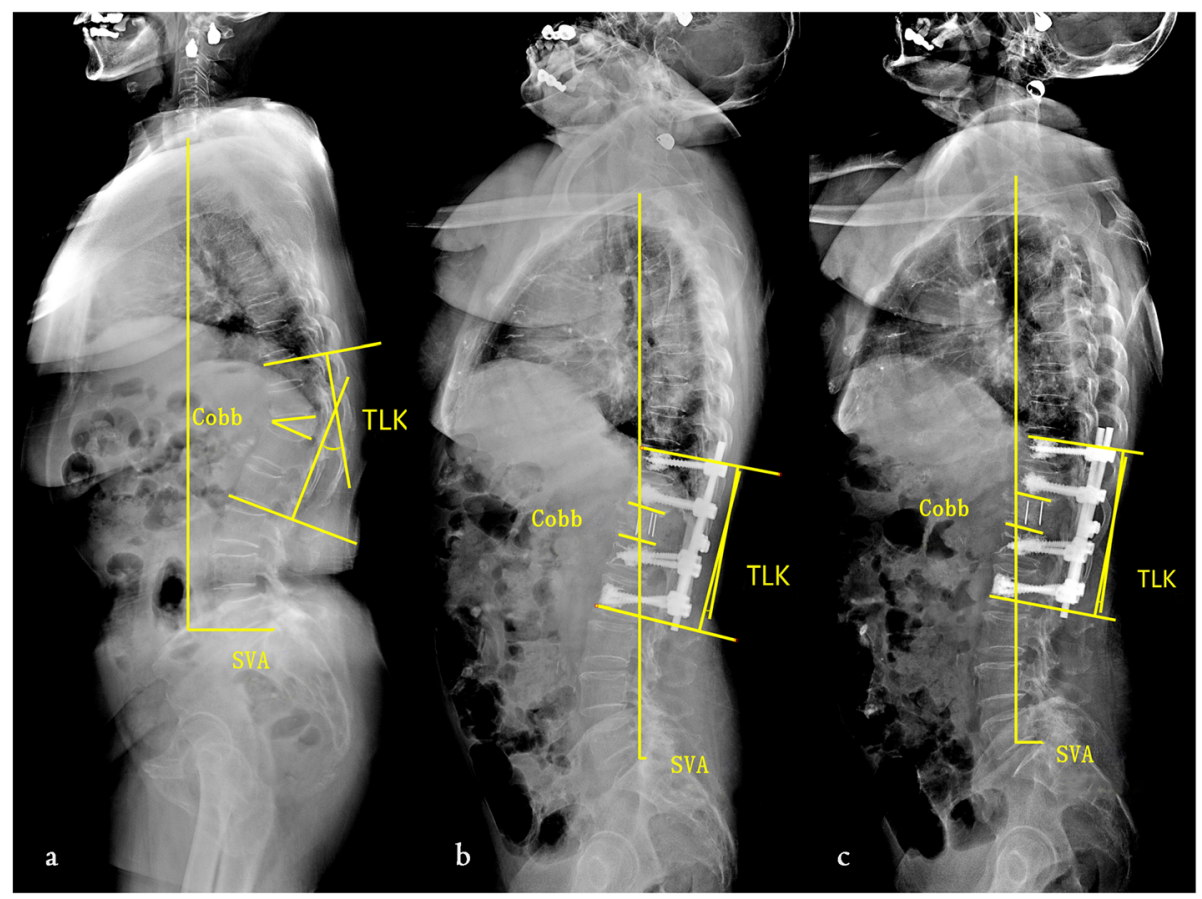

Fig. 3 The spinal sagittal parameters of a patient in group A

Our results suggest that in the surgical treatment for cases of kyphosis with no neurological deficits, it may also be important to pay attention to correcting the kyphosis and to regain a good balance of the sagittal plane. By doing so the abnormal stress on the articular process and intervertebral disc can be reduced and fatigue of the back muscles can also be reduced. So in group B, the focus of intraoperative treatment is the release of the intervertebral space on the side of the collapsing endplate of the injured vertebra. The purpose of loosening the gap is to correct local kyphosis and restore sagittal plane balance. Therefore, the bone-disc-bone osteotomy
(BDBO) was used in group B. Although BDBO belongs to Schwab IV osteotomy [32], it is not enough to remove part of the upper and lower endplates of the corresponding space to obtain a bone graft bed. This is because, besides the aim above, the range of BDBO should be as large as possible in order to get a spine which is easier to be corrected rather than focusing only on preparation of the bone graft bed. At the same time,after the pedicle screws were cemented, the increased holding force of the screw can increase the strength of the correction, achieve a satisfactory correction in the sagittal plane, and reduce the internal fixation segment.

Table 3 Comparison of data before and after operation and the last follow-up in group B

\begin{tabular}{|c|c|c|c|c|}
\hline Parameter & Before operation & After operation & Lastfollow-up & $P$ value \\
\hline$\overline{S V A}(\mathrm{~mm})$ & $62.5 \pm 26.6$ & $27.2 \pm 12.7$ & $30.7 \pm 11.6$ & 0.000 \\
\hline Cobb angle $\left(^{\circ}\right)$ & $26.5 \pm 7.3$ & $15.2 \pm 4.7$ & $15.7 \pm 4.6$ & 0.000 \\
\hline $\operatorname{TK}\left({ }^{\circ}\right)$ & $39.5 \pm 15.1$ & $32.1 \pm 14.5$ & $36.1 \pm 13.7$ & 0.462 \\
\hline $\operatorname{TLK}\left({ }^{\circ}\right)$ & $33.0 \pm 15.9$ & $7.3 \pm 1.3$ & $9.7 \pm 1.3$ & 0.000 \\
\hline $\mathrm{LL}\left({ }^{\circ}\right)$ & $48.4 \pm 14.9$ & $39.3 \pm 9.0$ & $42.0 \pm 9.3$ & 0.149 \\
\hline PT $\left({ }^{\circ}\right)$ & $17.7 \pm 8.4$ & $14.6 \pm 5.5$ & $15.1 \pm 4.4$ & 0.439 \\
\hline SS $\left(^{\circ}\right)$ & $24.9 \pm 9.5$ & $29.7 \pm 8.9$ & $26.5 \pm 10.5$ & 0.470 \\
\hline ODI & $44 \% \pm 8$ & & $19 \% \pm 5$ & 0.000 \\
\hline NRS & $4.0 \pm 1.1$ & & $0.8 \pm 0.6$ & 0.000 \\
\hline
\end{tabular}




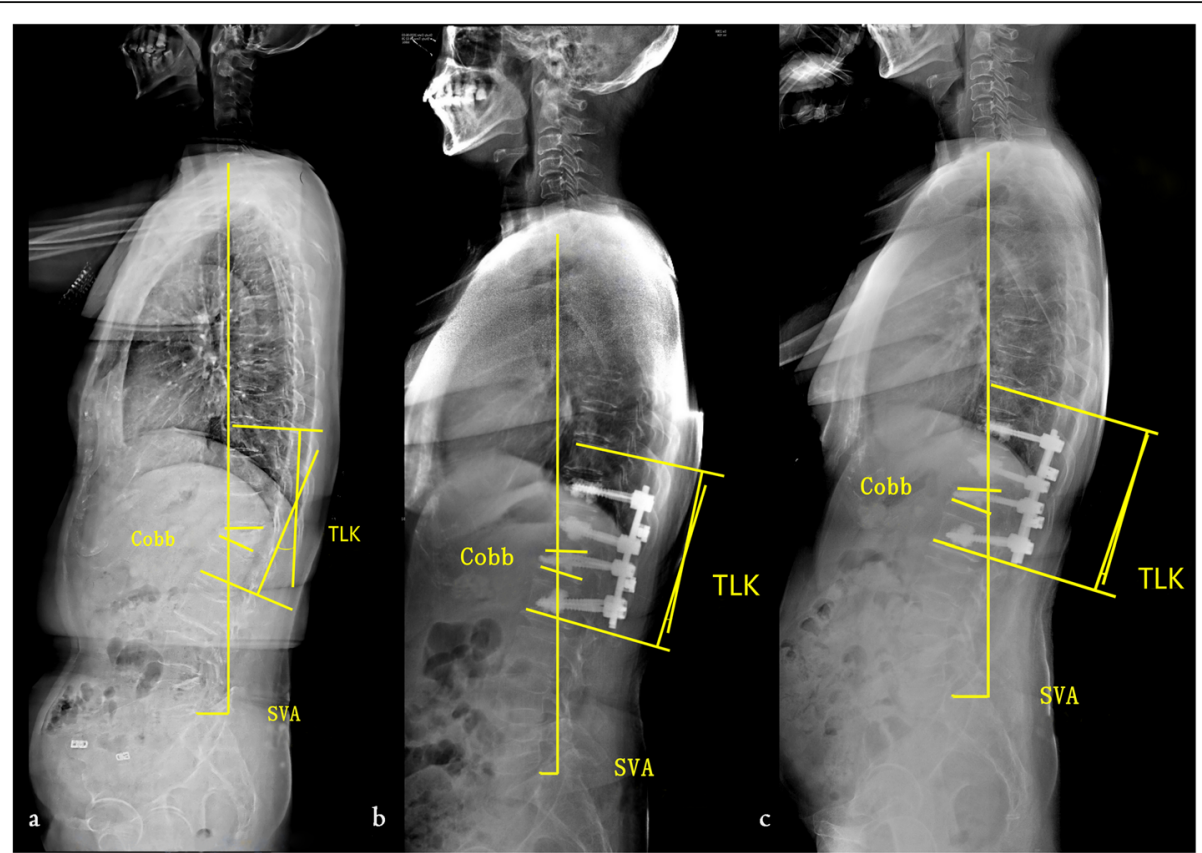

Fig. 4 The spinal sagittal parameters of a patient in group B

Table 4 Comparison of postoperative imaging results between group $A$ and $B$

\begin{tabular}{llll}
\hline Parameter & Group A & Group B & $P$ Value \\
\hline SVA $(\mathrm{mm})$ & $25.1 \pm 17.1$ & $27.2 \pm 12.7$ & 0.746 \\
Cobb angle $\left(^{\circ}\right)$ & $6.4 \pm 5.0$ & $15.2 \pm 4.7$ & 0.000 \\
TK $\left(^{\circ}\right)$ & $34.3 \pm 11.7$ & $32.1 \pm 14.5$ & 0.710 \\
TLK $\left(^{\circ}\right)$ & $5.7 \pm 3.3$ & $7.3 \pm 1.3$ & 0.140 \\
LL $\left(^{\circ}\right)$ & $34.4 \pm 7.5$ & $39.3 \pm 9.1$ & 0.190 \\
PT $\left(^{\circ}\right)$ & $20.6 \pm 7.8$ & $14.6 \pm 5.5$ & 0.047 \\
SS $\left(^{\circ}\right)$ & $35.6 \pm 9.9$ & $29.7 \pm 8.9$ & 0.156 \\
\hline
\end{tabular}

Table 5 Comparison of imaging and clinical results at the last follow-up between group A and B

\begin{tabular}{llll}
\hline Parameter & Group A & Group B & P Value \\
\hline SVA (mm) & $26.7 \pm 16.2$ & $30.7 \pm 11.6$ & 0.509 \\
Cobb angle $\left(^{(}\right)$ & $7.3 \pm 5.3$ & $15.7 \pm 4.6$ & 0.001 \\
TK $\left(^{\circ}\right)$ & $37.2 \pm 10.4$ & $36.1 \pm 13.7$ & 0.833 \\
TLK $\left(^{\circ}\right)$ & $7.1 \pm 3.2$ & $9.7 \pm 1.3$ & 0.014 \\
LL $\left(^{\circ}\right)$ & $38.4 \pm 5.0$ & $42.0 \pm 9.3$ & 0.284 \\
PT $\left(^{\circ}\right)$ & $22.6 \pm 8.2$ & $15.1 \pm 4.4$ & 0.013 \\
SS $\left(^{\circ}\right)$ & $36.5 \pm 9.2$ & $26.5 \pm 10.5$ & 0.028 \\
ODI & $23 \% \pm 5$ & $19 \% \pm 5$ & 0.073 \\
NRS & $0.9 \pm 0.3$ & $0.8 \pm 0.6$ & 0.700 \\
\hline
\end{tabular}


Table 6 Correlation analysis between clinical outcomes and sagittal parameters of group A

\begin{tabular}{|c|c|c|c|c|c|c|c|c|}
\hline & $\Delta S V A$ & $\Delta$ Cobb angle & $\Delta \mathrm{TK}$ & $\Delta \mathrm{TLK}$ & $\Delta \mathrm{LL}$ & $\Delta \mathrm{PI}$ & $\Delta \mathrm{PT}$ & $\Delta \mathrm{SS}$ \\
\hline \multirow[t]{2}{*}{$\Delta N R S$} & -0.363 & $0.773^{*}$ & 0.374 & 0.251 & 0.039 & -0.618 & $0.820^{*}$ & $-0.736^{*}$ \\
\hline & $(p=0.302)$ & $(p=0.009)$ & $(p=0.286)$ & $(p=0.485)$ & $(p=0.914)$ & $(p=0.057)$ & $(p=0.004)$ & $(p=0.015)$ \\
\hline \multirow[t]{2}{*}{$\Delta \mathrm{ODI}$} & 0.173 & -0.143 & 0.518 & -0.304 & -0.07 & -0.057 & 0.429 & -0.033 \\
\hline & $(p=0.632)$ & $(p=0.694)$ & $(p=0.125)$ & $(p=0.393)$ & $(p=0.838)$ & $(p=0.875)$ & $(p=0.217)$ & $(p=0.927)$ \\
\hline
\end{tabular}

*:p $<0.05, \triangle N R S$ improvement of NRS, $\triangle O D I$ improvement of ODI, $\triangle S V A$ change of SVA, $\triangle$ Cobb angle change of Cobb angle, $\triangle T K$ change of TK, $\triangle T L K$ change of TLK, $\triangle L L$ change of $\mathrm{LL}, \triangle P I$ change of $\mathrm{PI}, \triangle P T$ change of $\mathrm{PT}, \triangle \mathrm{SS}$ change of $\mathrm{SS}$

Table 7 Correlation analysis between clinical outcomes and sagittal parameters of group B

\begin{tabular}{lllllllll}
\hline & $\boldsymbol{\Delta}$ SVA & $\Delta$ Cobb angle & $\Delta$ TK & $\Delta$ TLK & $\Delta$ LL & $\Delta$ PI & $\Delta$ PT & $\Delta$ SS \\
\hline$\Delta$ NRS & 0.363 & $0.659^{*}$ & 0.269 & 0.345 & 0.309 & -0.205 & -0.103 & -0.026 \\
& $(P=0.246)$ & $(P=0.020)$ & $(P=0.398)$ & $(P=0.272)$ & $(P=0.329)$ & $(P=0.522)$ & $(p=0.749)$ & $(P=0.935)$ \\
$\Delta \mathrm{ODI}$ & 0.500 & -0.568 & -0.189 & -0.498 & 0.291 & -0.458 & -0.241 & 0.151 \\
& $(p=0.098)$ & $(p=0.054)$ & $(p=0.555)$ & $(p=0.099)$ & $\left(p^{-0.359)}\right.$ & $(p=0.134)$ & $(p=0.451)$ & $(p=0.639)$ \\
\hline
\end{tabular}

$*: p<0.05, \triangle N R S$ improvement of NRS, $\triangle O D I$ improvement of ODI, $\triangle S V A$ change of SVA, $\triangle$ Cobb angle change of Cobb angle, $\triangle T K$ change of TK, $\triangle T L K$ change of TLK, $\triangle L L$ change of $\mathrm{LL}, \triangle P I$ change of $\mathrm{PI}, \triangle P T$ change of $\mathrm{PT}, \triangle S \mathrm{~S}$ change of SS

The results of this study showed that the improvement of the NRS of the two groups was positively correlated with the improvement of the Cobb angle (local kyphosis) in the sagittal plane. In the case of group A, the improvement of NRS was also positively correlated with the change of PT but negatively correlated with the change of SS. It can be seen from the result that while the postoperative Cobb angle improved in the two groups, the local spine alignment was also restored accordingly. We also think that the improvement of the local alignment restored the stress on articular processes, intervertebral discs and other tissues to normal. At the same time, the improvement of the spinal alignment also reduced the work and strain of the back muscles, thereby relieving postoperative back pain. This result is basically consistent with the previous research [22, 23, 26, 31, 33]. In addition, it is considered that sagittal imbalance is an interactive phenomenon that is accompanied with alteration of LL, SS, PI and PT [34]. Therefore, once the sagittal balance is restored, the corresponding sagittal parameters will also change. We speculate that the surgical method of group A was more powerful than that of group B, which caused corresponding changes of PT and SS after the operation. However, the correlation between NRS and changes in PT, and SS needs to be further explored.

Our study had limitations. Firstly, due to the relatively small sample size and short follow-up time in this study the result may introduce some bias, and secondly, the long-term follow-up results of the above cases need to be further demonstrated.

\section{Conclusion}

For Kümmell's disease of thoracolumbar kyphosis with and without neurological deficits,to restore the normal spinal alignment and sagittal balance can obtain a satisfactory radiographic and clinical short and medium-term effects.

\section{Abbreviations}

SVA: Sagittal vertebral axis; TK: Thoracic kyphosis; TLK: Thoracolumbar kyphosis; LL: Lumbar lordosis; PI: Pelvic incidence; PT: Pelvic tilt; SS: Sacral slope; ODI: Oswestry disability index; NRS: Numerical rating scale; OVCF: Osteoporotic vertebral compression fracture; VCR: Vertebral column resection; BMD: Bone mineral density; ASIA: American spinal injury association; DVT: Deep vein thrombosis; CSF: Cerebrospinal fluid;

PJK: Proximal junctional kyphosis

\section{Acknowledgments}

We would like to thank Dr. Neale O. Haugen for his assistance with the professional linguistic editing. And thank Sheng Gao for her help in drawing.

\section{Authors' contributions}

JS and HC conceptualized and designed the study. HC drafted the initial manuscript. HC and GW carried out the analyses, reviewed and revised the manuscript. TL and XL reviewed and revised the manuscript. JS supervised the complete process of data collecting, and reviewed and revised the manuscript. All authors approved the final manuscript as submitted and agreed to be accountable for all aspects of the work.

Funding

There was no funding source for this research.

\section{Availability of data and materials}

The datasets generated and/or analyzed during the current study are not publicly available due to limitations of ethical approval involving the patient data and anonymity but are available from the corresponding author on reasonable request. 


\section{Declarations}

\section{Ethics approval and consent to participate}

This retrospective study was approved by the Medical Ethics Committee of Shandong Provincial Hospital and was conducted in accordance with the guidelines of the Declaration of Helsinki. Written informed consent was obtained from each patient.

\section{Consent for publication}

Not applicable.

\section{Competing interests}

The authors declare that they have no competing interests.

\section{Author details}

'Department of Spine Surgery, Shandong Provincial Hospital, Cheeloo College of Medicine, Shandong University, Jinan, Shandong, China. 2Department of Spine Surgery, Jinhua Municipal Central Hospital, Jinhua, Zhejiang, China. ${ }^{3}$ Department of Spine Surgery, Shandong Provincial Hospital Affiliated to Shandong First Medical University, Jinan, Shandong, China.

\section{Received: 24 February 2021 Accepted: 21 August 2021}

\section{Published online: 06 September 2021}

\section{References}

1. Steell HH. Kummell's disease. Am J Surg. 1951;81(2):161-7. https://doi.org/1 0.1016/0002-9610(51)90206-1.

2. Lee S-H, Kim ES, Eoh W. Cement augmented anterior reconstruction with short posterior instrumentation: a less invasive surgical option for Kummell's disease with cord compression. J Clin Neurosci. 2011;18(4):509-14. https:// doi.org/10.1016/j.jocn.2010.07.139.

3. Pius Kim, Seok Won Kim. Balloon Kyphoplasty: an effective treatment for Kummell disease? Korean J Spine 2016;13(3):102-106, DOl: https://doi.org/1 0.14245/kjs.2016.13.3.102

4. Yan-Sheng Huang, Chao-Yuan Ge, Hang Feng et al. Bone cementaugmented short-segment pedicle screw fixation for Kümmell disease with Spinal Canal stenosis. Med Sci Monit 2018;14(24):928-935.

5. Mei L, Sang W, Chen Z, Lou C, Lin Z, Jin K, et al. Titanium mesh bone grafting combined with pedicle screw internal fixation for treatment of Kummell disease with cord compression a case report and literature review. Medicine (Baltimore). 2018;97(36):e12183. https://doi.org/10.1097/MD. 0000000000012183.

6. Nickell LT, Schucany WG, Opatowsky MJ. Kummelldisease. Proc (Baylor Univ Med Cent). 2013:26(3):300-1.

7. Lim J, Choi S-W, Youm J-Y, Kwon H-J, Kim S-H, Koh H-S. Posttraumatic delayed vertebral collapse: Kummell's disease. J Korean Neurosurg Soc 2018;61(1):1-9. https://doi.org/10.3340/jkns.2017.0505.010

8. H Li, C Z Liang, Q X Chen. Kümmell's Disease,an Ucommon and Complicated Spinal Disorder: a Review. J Int Med Res 2012; 40: 406-414

9. Wang F, Wang D, Tan B, et al. Comparative Study of Modified Posterior Operation to Treat Kümmell's Disease. Medicine (Baltimore). 2015:94(39):e1595.

10. Karin Swartz, Dominic Fee. Kümmell's disease a case report and literature review. Spine 2008:33(5):E152-E155.

11. Lee SH, Jain V, Lee S-H, Baek OK, Moon KH. Transpedicular intrabody cage insertion with posterior spine stabilization in Kümmell disease: a report of two cases. World Neurosurg. 2018;116:236-40. https://doi.org/10.1016/j. wneu.2018.05.133.

12. Rajasekaran S, Rajoli SR, Aiyer SN, et al. A Classifification for kyphosis based on column Defificiency, curve magnitude, and osteotomy requirement. J Bone Joint Surg Am. 2018;100(13):1147-56. https://doi.org/10.2106/JBJS.17.01127.

13. Zhang $X, \mathrm{Hu}$ W, Yu J, et al. An effective treatment option for Kümmell's disease with neurological deficits: Modified transpedicular subtraction and disc osteotomy combined with long-segment fixation. Spine. 2016;41(15):E923-30.

14. Yongjae Cho. Posterior vertebrectomy and circumferential fusion for the treatment of advanced Thoracolumbar Kümmell Disease with Neurologic Deficit. Asian Spine J 2017;11(4):634-640i.

15. Yang DL, Yang SD, Chen Q, et al. The Treatment Evaluation for Osteoporotic Kummell Disease by Modified Posterior Vertebral Column Resection: Minimum of One-Year Follow-Up. Med Sci Monit. 2017;23:606-12.
16. Feng Yu Liu, Li Shuang Huo, Sen Liu, et al. Modifified posterior vertebral column resection for Kümmell disease. Medicine (Baltimore) 2017;96(5): e5955.

17. Wang H, Ding W. Posterior vertebral column resection through unilateral osteotomy approach for old lumbar fracture combined with Kummell's disease: A case report. World Neurosurg. 2018;109:147-51.

18. Wang W, Liu Q, Liu W-j, Li Q-b, Cai L, Wang Z-k. Different performance of Intravertebral vacuum clefts in Kümmell's disease and relevant treatment strategies. Orthop Surg. 2020;12(1):199-209. https://doi.org/1 0.1111 /os.12609.

19. Yu H, Li Y, Yao X, Lin J, Pan Y, Zhuang H, et al. Application of percutaneous vertebroplasty and percutaneous kyphoplasty in treating Kümmell's patients with different stages and postural correction status. Exp Rev Med Devices. 2020;17(4):357-64. https://doi.org/10.1080/17434440.2020.1736039.

20. Lee K, Adsul N, Kim H-S, et al. Percutaneous pedicle screw fixation with bone cement augmentation under epidural anesthesia for the treatment of Kümmell's disease at extremely old age: a case report. World Neurosurg. 2018;119:506-10. https://doi.org/10.1016/j.wneu.2018.06.061.

21. Bing $Q$, Dingjun $H$, Zheng $Y$, et al. Effectiveness comparison between two operations in treatment of unstable type Kümmell's disease. Zhongguo Xiu Fu Chong Jian Wai Ke Za Zhi. 2017;31(2):185-90.

22. Lamartina C, Berjano P. Classifification of sagittal imbalance based on spinal alignment and compensatory mechanisms. Eur Spine J. 2014;23(6):1177-89. https://doi.org/10.1007/s00586-014-3227-9.

23. Tae Sik Goh,Jong Ki Shin,Myung Soo Youn, et al. Surgical versus nonsurgical treatment of lumbar degenerative kyphosis. Eur Spine J 2017;26(8):21532159, DOl: https://doi.org/10.1007/s00586-017-5008-8.

24. Xia W, Han F, Zhu Z, et al. Association between back muscle degeneration and spinal-pelvic parameters in patients with degenerative spinal kyphosis. BMC Musculoskelet Disord. 2019;20(1):454. https://doi.org/10.1186/s12891-01 9-2837-0

25. Xia W, Liu C, Duan S, Xu S, Wang K, Zhu Z, et al. The influence of spinalpelvic parameters on the prevalence of endplate Modic changes in degenerative thoracolumbar/lumbar kyphosis patients. PLoS One. 2018; 13(5):e0197470. https://doi.org/10.1371/journal.pone.0197470.

26. Hori Y, Matsumura A, Namikawa T, et al. Does sagittal imbalance impact the surgical outcomes of shortsegment fusion for lumbar spinal stenosis associated with degenerative lumbar scoliosis? J Orthop Sci. 2019;24(2):2249. https://doi.org/10.1016/j.jos.2018.10.005.

27. Masaki M, Ikezoe T, Yanase K, et al. Association of Pain History and Current Pain with Sagittal Spinal Alignment and muscle stiffness and muscle mass of the Back muscles in middle-aged and elderly women. Clin Spine Surg. 2019;32(7):E346-52. https://doi.org/10.1097/BSD.0000000000000793.

28. Z.-K. Duan, J.-F. Zou, X.-L. He, et al. Bone-filling mesh container versus percutaneous kyphoplasty in treating Kümmell's disease. Arch Osteoporos 2019:14(1):109, DOI: https://doi.org/10.1007/s11657-019-0656-4.

29. Mazel C, Ajavon L. Malunion of post-traumatic thoracolumbar fractures. Orthop Traumatol Surg Res. 2018;104(1S):S55-62. https://doi.org/10.1016/j. otsr.2017.04.018

30. Bernhardt M, Bridwell KH. Segmental analysis of the sagittal plane alignment of the normal thoracic and lumbar spines and thoracolumbar junction. Spine. 1989;14(7):717-21. https://doi.org/10.1097/00007632-1 98907000-00012.

31. Glassman SD, Bridwell K, John R. Dimar, et al. The impact of positive sagittal balance in adult spinal deformity. Spine. 2005;30(18):2024-9. https://doi. org/10.1097/01.brs.0000179086.30449.96.

32. Schwab F, Blondel B, Chay E, et al. The comprehensive anatomical spinal osteotomy classification. Neurosurgery. 2014;74(1):112-20 discussion 120.

33. He S, Zhang Y, Ji W, et al. Analysis of Spinopelvic Sagittal Balance and Persistent Low Back Pain (PLBP) for Degenerative Spondylolisthesis (DS) following Posterior Lumbar Interbody Fusion (PLIF). Pain Res Manag. 2020; 2020:5971937.

34. Labelle H, Roussouly P, Berthonnaud E, et al. Spondylolisthesis, pelvic incidence, and spinopelvic balance. Spine. 2004;29(18):2049-54. https://doi. org/10.1097/01.brs.0000138279.53439.cc.

\section{Publisher's Note}

Springer Nature remains neutral with regard to jurisdictional claims in published maps and institutional affiliations. 